Voix et Images

\title{
Impasses ou issues? L'imaginaire masculin face à la femme
}

\section{Patricia Smart}

Volume 12, numéro 3 (36), printemps 1987

Yves Beauchemin

URI : https://id.erudit.org/iderudit/200671ar

DOI : https://doi.org/10.7202/200671ar

Aller au sommaire du numéro

\section{Éditeur(s)}

Université du Québec à Montréal

\section{ISSN}

0318-9201 (imprimé)

1705-933X (numérique)

Découvrir la revue

\section{Citer cet article}

Smart, P. (1987). Impasses ou issues? L'imaginaire masculin face à la femme.

Voix et Images, 12(3), 555-560. https://doi.org/10.7202/200671ar d'utilisation que vous pouvez consulter en ligne.

https://apropos.erudit.org/fr/usagers/politique-dutilisation/ 


\title{
Impasses ou issues? L'imaginaire masculin face à la femme
}

\author{
par Patricia Smart, Université Carleton
}

Décidément il n'est pas facile d'être un homme! Voilà l'impression globale que j'ai eue en lisant deux parutions récentes d'hommes où la question du rapport à la femme et au féminin sous-tend - consciemment ou inconsciemment - toute la réflexion sur l'écriture, la pensée, la vie. Dans le dernier roman d'Yvon Rivard, les Silences du Corbeau, la symbolique patriarcale du féminin est traversée avec une légèreté et un humour qui n'excluent pas la profondeur; au contraire, le bon sens teinté de scepticisme de son narrateur permet à Rivard de s'approcher allègrement du gouffre féminin-maternel qui a happé tant d'écrivains québécois avant lui. Dans A Double sens: échanges sur quelques pratiques modernes de Hugues Corriveau et Normand de Bellefeuille, la même traversée du langage et de l'ordre symbolique se fait à grands coups de théorie, et la rencontre du féminin se fait comme à contre-cœur (à contre-tête?); mais elle se fait, et elle est transformatrice. Sur la scène postmoderne masculine, il semble que tout doive passer d'abord par la tête: l'enjeu c'est d'en sortir. 
Les silences du Corbeau ${ }^{1}$ rappelle par plusieurs biais les romans de Jacques Godbout et d'Hubert Aquin, dont Rivard est à n'en pas douter le fils spirituel. Histoire d'un groupe de Québécois réunis en compagnie de quelques étrangers dans un ashram en Inde, c'est l'Aquarium des années 1980, mais avec l'humour de Salut Galarneau et une fascination de la coïncidence des contraires qui rappelle plutôt l'imaginaire aquinien. Alexandre, le narrateurprotagoniste, confie ses réflexions au fil des jours à son journal, et écrivant dans sa petite chambre il fait penser au narrateur emprisonné de Prochain épisode:

Le corbeau passait et repassait devant la fenêtre de mon cachot, en effleurant les barreaux du bout de ses ailes. J'étais là depuis des années, des siècles, plus personne ne se souvenait de moi, de cette prison, et moi-même je ne me souvenais plus de celui qui avait été incarcéré.

Et quel merveilleux mélange de Godbout-Aquin dans le passage où, ayant tiré l'hexagramme "Le feu dans le lac" du Yi King, Alexandre se trouve transporté par le souvenir vers un "moment de vérité» de son enfance: le tranquille bonheur d'une fin de journée de pêche où debout sur un rocher il a éprouvé une sensation d'attente infinie face à ce... lac incendié par le feu plus vif du soleil couchant (p. 26). Mais à la différence d'Aquin, qui s'épuisait dans une sorte de rivalité masculine visant à conquérir le vide et à pénétrer son mystère, Rivard est capable d'écoute, d'attention, d'assez d'humilité (ou de confiance) pour commencer à déconstruire cet appareil mystifiant dont la féminité a toujours été entourée dans la tête des hommes. Ce qui débouche nécessairement sur une reconnaissance de la femme réelle...

Pour Alexandre et ses compagnons, l'énigme s'incarne dans le personnage de Mère, la jeune Indienne qui sert de "divinité en résidence» à l'ashram, et qui les reçoit silencieusement chaque jour dans une cérémonie teintée d'érotisme sacré. Représentante de l'Autre féminin, Mère est l'insondable "absente" qu'il s'agit de cerner et de séduire afin que l'identité masculine soit assurée. Rivard décrit avec une ironie délicieuse tous ces hommes - un peintre visionnaire, un romancier-poète-essayiste... qui se spécialise dans l'extase, un mangeur de yaourt en proie à un adversaire invisible - et les quelques femmes qui leur tiennent passivement compagnie. Échoués en Inde après leurs différents échecs, tous sont à la recherche du divin, des secrets de la représentation, d'une maîtrise intellectuelle du gouffre intérieur qui les tourmente; et tous ignorent, fuient ou exploitent les femmes réelles dans leur entourage. Si Alexandre se différencie des autres, c'est par un sain scepticisme qui l'empêche de croire que tout peut se résoudre à l'intérieur des parois de sa tête. Pourtant lui aussi est en fuite, d'une épouse et d'une amante entre lesquelles il n'arrive pas à "choisir».

Cette allégorie transparente permet à Rivard d'arpenter les contradictions d'un imaginaire masculin qui semble mâ jusque dans ses pulsions les plus profondes par un besoin de rivalité et de domination. Descártes, l'Oedipe, le père castrateur, l'impérialisme, tout y passe; et toujours, en toile de fond aux 
analyses interminables dans lesquelles les personnages tournent en rond, insiste la presque insurmontable difficulté ressentie par l'homme de s'engager dans un véritable rapport avec la femme: Je ne suis au fond qu'une caricature de Faust, écrit Alexandre, je veux tout connaître sans signer aucun pacte (p.32). Malgré sa douceur, Alexandre est harcelé dans ses rêves par des images de meurtre et de viol, comme si, incapable de dissocier l'amour de la possession, il est condamné à rêver les femmes qu'il aime livrées à d'autres désirs que le sien: N'est-ce pas pour cela, pour ne pas avoir à partager mon plaisir ou à en reconnaître la violence, que je les imaginais tantôt innocentes tantôt coupables? (p. 169).

Que «la Femme» dans sa toute-puissance castratrice n'est qu'une création de l'homme est merveilleusement suggéré par la figure paternelle du mesquin Chitkara qui se cache derrière la divinité de Mère et manipule son image à son propre profit. Toutefois, l'importance du roman de Rivard tient peut-être moins dans cette démystification du symbole Mère que dans la lucidité de son regard sur le "comment vivre" et le "comment écrire" qui en résultent pour le sujet masculin. Comment vivre sans réduire l'autre à un reflet de soi; comment écrire sans tuer? Rappelant son propre père qui march [ait] en raquettes dans un champ recouvert de neige sans y laisser de traces (p. 192), Alexandre se situe dans un rapport de continuité avec la symbolique masculine québécoise (cf. Menaud, qui, lui, se définissait «maître» par l'empreinte laissée par ses raquettes). Mais il s'en libère en même temps par sa volonté d'habiter le silence et le réel autrement que par la conquête. Pour ce faire, suggère Rivard dans un très beau passage, les hommes devront se mettre d'abord à une véritable écoute des paroles et des silences des femmes:

Pourquoi les hommes sont-ils incapables d'un tel silence? Parfois je pense que seules les femmes ont réussi à naître sans déchirer la nuit qui nous enveloppe et qu'ainsi la mort, dont nous croyons nous éloigner à la vitesse de la parole, leur sera plus naturelle qu'à nous. Pensée sexiste? Peut-être. N'empêche que j'aimerais être femme pour avoir accès à ce qui se trame devant et derrière moi, pour pouvoir enfin, autrement qu'en pensée, être et ne pas être.

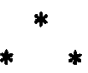

Lisant $\bar{A}$ Double Sens: échanges sur quelques pratiques modernes ${ }^{2}$ immédiatement après les Silences du Corbeau, je n'ai pu m'empêcher de reconnaître une certaine parenté entre ses auteurs et les habitants tourmentés, impuissants, mais jamais à court de mots de l'ashram de Rivard. Sans avoir quitté le territoire québécois, Corriveau et de Bellefeuille sont aussi sûrement en exil que les personnages de Rivard, mais l'espace de leur exil s'identifie au paysage post-moderne. Seuls, vieillissants, fatigués, la tête remplie à craquer de théorie française, ils exécutent une triste danse à travers les débris d'une pensée moderne acculée au spectacle de sa propre stérilité. Et le fait qu'ils savent tout, qu'ils balaient tout par la lucidité de leur regard, ne rend que plus triste leur apparente impasse. 
Et pourtant la puissance transformatrice de ce livre - sa "séduction", pour employer un mot qui préoccupe beaucoup les auteurs - tient précisément dans l'atmosphère irrespirable de cet étalement de connaissances théoriques, sorte de forteresse de l'esprit dans laquelle petit à petit, et comme bloquées de toutes parts, s'infiltrent des traces' du féminin, de la vie, du réel. Déjà au début de leur trajet, Corriveau et de Bellefeuille définissent leur projet comme une tentative de situer leur écriture dans ce qu'elle a de spécifiquement masculin. Comme Rivard, ils savent qu'il y a du masculin (p. 13) dans leur obsession de l'écriture comme un moyen de détourner la mort, celle qui nous ferait nous taire (p. 12), et ils reconnaissent chez les femmes une façon différente de questionner la mort et le silence: Mais ne serait-ce pas... une façon solidaire d'être avec elles que de reconnaître cette différence dans la manière de questionner le désastre? (p. 15).

Sont-ils conscients, tout de même, du caractère obsessionnel de cette identité masculine qui sous-tend leurs réflexions sur la modernité (qui était pourtant un projet conçu pour échapper à la vieille «métaphysique» fondée dans l'identité)? On se le demande, car une part d'ironie, de "construit", se cache derrière l'apparente spontanéité de ces lettres écrites sur une période de dix-huit mois. Réfléchissant au paradoxe d'une modernité maintenant vieille de quinze ans, ils avouent la peur d'être remplacés (Il se pourrait qu'un certain effet 'Hexagone' nous guette, p. 18), le soupçon qu'ils sont devenus une institution ou un monument, et ceci sans avoir changé quoi que ce soit dans l'ambiance idéologique de leur temps. Étant donné le désir précédemment mentionné de se situer par rapport au féminisme, il est étonnant de lire chez eux tel constat désabusé: l'horizon révolutionnaire [est] maintenant bouché, les illusions d'une praxis révolutionnaire et totale [se sont] estompées... (p. 22). C'est à croire que la praxis féministe, pourtant vraiment transformatrice du discours patriarcal, n'a pas encore "pénétré» la conscience de ces "désastrés" post-modernes, pour qui la voix du Père-Censeur exerce encore son autorité à travers l'intertexte de la théorie française.

L'effet de censure joué par la théorie se révèle du début à la fin du volume, et là encore les auteurs - toujours conscients de ce qu'il y a de théâtral dans leur démarche - en révèlent une certaine conscience: $\mathrm{De}$ la 'tradition', dis-tu, et la 'citation', elle, n'en témoignerait-elle pas d'une certaine façon? (p. 25). Les citations auxquelles ils ont recours pour renforcer l'autorité de leur propre parole ont paradoxalement l'effet de couper cette parole, comme dans ce passage où de Bellefeuille, sur le point de formuler clairement les dilemmes de la modernité, s'interrompt lui-même pour chercher une citation convenable(!):

[ Il nous faudrait] une modernité qui, n'ayant plus à pa(e)nser les plaies d'un échec, peut dorénavant inventer... sa propre praxis, non plus seulement parodique mais véritablement transformatrice... Je cherche donc une citation intelligente sur la postmodernité...

Et à partir de ce moment, égaré dans ses citations, il semble oublier le souci de transformation dont il était si proche. 
Tout ceci ne serait-il qu'un énorme jeu pratiqué aux dépens du lecteur, une auto-parodie de l'impuissance post-moderne? Ce qui est certain, c'est que devant ce texte si plein (si rempli), si hyperconscient, je me suis sentie poussée vers une position non pas de partage ou d'échange, mais de rivalité et presque de contre-agression: modalités masculines du rapport au texte qui semblent régies par la stratégie de "feintes» et de demi-révélations adoptées par les auteurs. Intéressant, dans ce livre qui porte le titre d' "Echanges», ce passage où les auteurs occultent la place normalement réservée au lecteur pour la remplir par eux-mêmes:

Il y a toujours, idéalement, cette troisième personne au livre, 'l'autre qui vient troubler la séance'. C'est qu'ici simplement elle a 'nom' et 'style' et qu'il nous faut compter chaque fois avec la 'signature' de cette 'troisième donnée'. Nous sommes nous-mêmes l'autre, habituellement innommable, dans le texte.

Ainsi me suis-je mise (agressivement?) à remarquer l'ironie d'une situation qui échappe aux deux auteurs. C'est d'une part leur fuite éperdue en avant pour échapper à ce qu'ils appellent le piège du passé (p. 29), l'angoisse qu'une certaine tradition nous a trop bien montré à...investir [dans l'écriture ] (p. 35). Et d'autre part, tandis que par leur tourment, leur sentiment du devoir (Il faudrait que..., Nous ne pouvons pas nous permettre de..., etc.), leur vulnérabilité et leur honnêteté foncière (Car ne faut-il pas... de l'assurance pour charmer? Et l'écriture encore m'inquiète trop, peut-être, pour que mon texte me séduise, p. 11): autant de marques, autant d'indices d'un discours québécois auquel la modernité a tenté d'échapper par une rupture brutale.

Rejetant leurs pères littéraires québécois, Corriveau et de Bellefeuille semblent préférer être les fils impuissants d'une pensée française (LyotardFelman-Deleuze-Genette etc.) qui les empêche de trouver leurs propres modalités d'écrire, de penser et de vivre. Et pourtant, cernés de toutes parts par des miroirs, des étiquettes, du déjà-et-mieux-dit, menacés par [leurs] propres textes de perdre la face (p.23), c'est avec leurs prédécesseurs masculins québécois qu'ils renouent à leur insu. N'est-ce pas Saint-Denys Garneau qui - cinquante ans plus tôt - avait avoué dans des mots presque identiques aux leurs son impuissance devant un langage qu'il concevait comme «ennemi»?

\section{Parole sur ma lèvre déjà prends ton vol. tu n'es plus à moi \\ Va-t'en extérieure, puisque tu l'es déjà ennemie... \\ Impuissant sur toi maintenant dès ta naissance ${ }^{3}$}

Et Hubert Aquin, vingt ans avant eux: ...je gagne quelque chose à ce jeu, je gagne du temps: un temps mort que je couvre de biffures et de phonèmes, que j'emplis de syllabes et de hurlements... ${ }^{4}$. A la place de ces écrits québécois qu'ils ne citent nulle part dans leur correspondance, Corriveau et de Bellefeuille substituent des citations françaises qui en sont le fidèle écho, telle cette phrase de Cioran: Nous mourons en proportion des mots que nous jetons tout autour de nous (p. 40). 
N'y a-t-il donc pour l'homme d'autre échappatoire à la mort qui l'obsède que ce remplissage frénétique du vide par une accumulation de mots? $\mathrm{Tu}$ décolles, je détourne, nous écrivons bien en effet et c'est précisément ce vide qui t'inquiète que page à page nous piégerons (p. 15 , je souligne): et à michemin du trajet, ce constat de notre indissimulable plaisir devant la quantité, devant le volume palpable du livre qui se construit (p. 133). L' "anxiété de l'influence» dont parle $\mathrm{H}$. Blum doit-elle toujours enfermer les fils dans une révolte/répétition de la situation de leurs pères?

Comme dans le roman de Rivard, la sortie se situe du côté d'un féminin confiné aux marges du texte, mais qui insiste, encore et encore, jusqu'à ce qu'il infiltre les propos des auteurs et les rende enfin respirables. Un exemple: la discussion de «La Marche» de France Théoret, un texte qui contient le genre de "rythme pulsionnel» kristévien, "maternel», dont les auteurs reconnaissent (cérébralement) l'importance, mais qu'ils tuent presque littéralement par la lourdeur de l'appareil critique qu'ils y appliquent. Qu'est-ce qui animerait le rythme de ce texte-là, demandent-ils (p. 82); et de là à y compter 224 mots de 4 lettres,.. 62 mots de 8 lettres et 8 mots de 12 lettres: ce qui donne 294 mots qui sont directement liés à ce 4 fondamental (p. 82), il n'y a (pour eux) qu'un pas. Mais si c'était le mouvement, la vie, le féminin qui animaient ce texte? Un féminin qui échappera toujours aux catégories masculines? Mais je m'emporte, j'écris trop moi-même, et dans mon indignation j'oublie presque de parler de phrases apparemment anodines, comme le souhait de de Bellefeuille de créer... un trou d'air d'où surgirait autre chose que cela qui est bien dit (p. 122), qui prennent de plus en plus de place dans le texte et le tirent du côté de la vie. Corriveau et de Bellefeuille finissent par déjouer la censure des Pères français qui les tiennent en laisse, par l'aveu impitoyablement honnête de leur ennui, leur peur, leur épuisement, dans cette rivalité qui surgit malgré eux et qu'ils vainquent par l'amitié. Et surtout dans leurs propres poèmes qui respirent malgré tout, malgré la dissection à laquelle les ont livrés leurs auteurs. Ironiquement, c'est par leur souhait un peu triste de croire à quelque chose malgré tout ce qu'ils savent, qu'ils finissent par séduire, comme ils ont souhaité le faire tout au long de leur périple. Je ne pourrai sans doute jamais dire que j'écris pour; mais... (p. 132), écrit l'un d'entre eux. Les points de suspension laissent-ils espérer un "prochain épisode" à la modernité masculine québécoise? Qui serait un véritable dialogue avec les femmes et une écoute du féminin?

1. Montréal, Éditions du Boréal-Express, 1986.

2. Montréal, les Herbes rouges, 1986.

3. Poésies complètes, Montréal, Fides, 1949, p. 123.

4. Prochain épisode, Montréal, le Cercle du Livre de France, 1965, p. 13. 